\section{PANDAS (Pediatric Autoimmune Neuropsychiatric Disorders Associated With Streptococcal Infection): A Case Report}

To the Editor: The prepubertal onset of obsessive-compulsive disorder (OCD), Tourette's syndrome, or tic disorder with abrupt symptom exacerbation after streptococcal infection has been termed PANDAS (pediatric autoimmune neuropsychiatric disorders associated with streptococcal infection).

\section{Case Report}

A 13-year-old boy was seen for acute onset of exuberant odd movements of the body and arms, associated with vocalizations, and worsening of his previous simple motor tics of the head and neck. He had a family history of simple motor tics (father and maternal uncle) and personal history of repeated throat infections during childhood documented by raised anti-streptolysin-O antibodies titers. During inpatient care he presented with obsessive-compulsive symptomatology (e.g., intrusive thoughts related to death and repeated saying of the word "no" that relieved them) and simple motor tics (eye blinking and neck jerking) that worsened in anxiety-producing situations. He also claimed that the odd movements described above relieved his death-related intrusive thoughts. A brain MRI showed no abnormality, anti-streptolysin-O antibodies titer was normal, and his total IQ score was 76 .
Previous Neuropsychiatric History At the age of 9, the patient had acutely developed intrusive thoughts of contamination and repeated hand washing associated with simple motor (eye blinking and neck jerking) and vocal (throat clearing) tics. A throat infection was documented at that time with raised anti-streptolysin-O antibodies titer. He was medicated with sertraline, $50 \mathrm{mg}$ q.i.d., risperidone, $2 \mathrm{mg}$ q.i.d., and penicillin, 2,000 units per month during 6 months. Two weeks later a significant symptomatic improvement had occurred. During the following waxing and waning course of his disease, he never experienced vocal tics again. Some of the simple motor tics and obsessive-compulsive symptom exacerbations occurred after a throat infection, were documented by raised antistreptolysin- $\mathrm{O}$ antibodies titers, and were treated with penicillin.

A symptomatic remission occurred with $150 \mathrm{mg}$ of fluvoxamine, $5 \mathrm{mg}$ of haloperidol and 2 $\mathrm{mg}$ of biperiden. These doses were progressively titrated.

\section{Discussion}

Our patient had an abrupt onset of a tic disorder (most probably Tourette's syndrome) and OCD, with a waxing and waning course, and symptomatic exacerbations temporally related to group A betahemolytic streptococcus (GABHS) infection. A long history of repeated throat infections preceded the onset of the disease. This clinical picture fulfills current proposed criteria for the diagnosis of PANDAS. $^{1}$

The diagnosis and existence of PANDAS are controversial. There are some data supporting the theory that it is the result of an auto-immune-mediated process, in which anti-streptolysin-O antibodies are thought to be involved, affecting the basal ganglia. ${ }^{2,3}$

Tic disorders respond to a variety of pharmacologic agents (e.g., antipsychotics). OCD is best treated with a combination of medication (typically an SSRI) and cognitive behavior therapy. Antibiotics are indicated only for the treatment of acute streptococcal infections.

Immunomodulatory therapies may be considered for severely affected children. ${ }^{1}$

Luís Fonseca, M.D.

Psychiatry and Mental Health Department, S. Marcos Hospital, Braga, Portugal

JoÃo Guerra, M.D.

Child and Adolescent Psychiatry Department, Porto Hospital Centre, Porto, Portugal

Nuno Neves, M.D.

Child and Adolescent Psychiatry Department, Infante D.

Pedro Hospital, Aveiro, Portugal

Álvaro Machado, M.D.

Neurology Department, S. Marcos Hospital, Braga, Portugal

\section{References}

1. Swedo SE, Leonard HL, Rapoport JL: The pediatric autoimmune neuropsychiatric disorders associated with streptococcal infection (PANDAS) subgroup: separating fact from fiction. Pediatrics 2004; 113:907-911

2. Leslie DL, Kozma L, Martin A, et al: Neuropsychiatric disorders associated with streptococcal infection: a case control study among privately insured children. J Am Acad Child Adolesc Psychiatry $2008 ; 47: 1166-1172$

3. Hoffman KL, Hornig M, Yaddanapudi K, et al: A murine model for neuropsychiatric disorders associated with group A 


\section{LETTERS}

$\beta$-hemolytic streptococcal infection.

J Neurosci 2004; 24:1780-1791

4. Miller DL, Laxer RM: Pediatric autoimmune neuropsychiatric disorders associated with streptococcal infections. Pedi- atr Rheumatol Online J 2003; vol 1. Available at http://www.pedrheum onlinejournal.org/April/reviewarti.htm

5. Kurlan R, Kaplan EL: The pediatric autoimmune neuropsychiatric disorders asso- ciated with streptococcal infection (PANDAS) etiology for tics and obsessive-compulsive symptoms: hypothesis or entity? Practical considerations for the clinician. Pediatrics 2004; 113:883-886 